\title{
Overexpression of PpSnRK1a in tomato enhanced salt tolerance by regulating $A B A$ signaling pathway and reactive oxygen metabolism
}

Wen-Ru Wang, Jia-Hui Liang, Gui-Fang Wang, Mao-Xiang Sun, Fu-Tian Peng ${ }^{*}$ and Yuan-Song Xiao*

\begin{abstract}
Background: SNF-related Kinase 1 (SnRK1) is a key component of the cell signaling network. SnRK1 is known to respond to a wide variety of stresses, but its exact role in salt stress response and tolerance is still largely unknown.

Results: In this study, we reported that overexpression of the gene encoding the a subunit of Prunus persica SnRK1 (PpSnRK1a) in tomato could improve salt stress tolerance. The increase in salt stress tolerance in PpSnRK1aoverexpressing plants was found to correlate with increased PpSnRK1a expression level and SnRK1 kinase activity. And PpSnRK1a overexpression lines exhibited a lower level of leaf damage as well as increased proline content and reduced malondialdehyde (MDA) compared with wild-type (WT) lines under salt stress. Furthermore, PpSnRK1a enhanced reactive oxygen species (ROS) metabolism by increasing the expression level of antioxidase genes and antioxidant enzyme activities. We further sequenced the transcriptomes of the WT and three PpSnRK1a overexpression lines using RNA-seq and identified about 1000 PpSnRK1a-regulated genes, including many antioxidant enzymes, and these genes were clearly enriched in the MAPK signaling pathway (plant), plant-pathogen interactions and plant hormone signaling transduction and can respond to stimuli, metabolic processes, and biological regulation. Furthermore, we identified the transcriptional levels of several salt stress-responsive genes, SIPP2C37, SIPYL4, SIPYL8, SINAC022, SINAC042, and SISnRK2 family were altered significantly by PpSnRK1a, signifying that SnRK1a may be involved in the ABA signaling pathway to improve tomato salt tolerance. Overall, these findings provided new evidence for the underlying mechanism of SnRK1a conferment in plant salt tolerance phenotypes.
\end{abstract}

Conclusions: Our findings demonstrated that plant salt stress resistance can be affected by the regulation of the SnRK1a. Further molecular and genetic approaches will accelerate our knowledge of PpSnRK1a functions, and inform the genetic improvement of salt tolerance in tomato through genetic engineering and other related strategies.

Keywords: PPSnRK1a, ROS metabolism, ABA signaling, Salt tolerance

\footnotetext{
*Correspondence: pfruit@163.com; ysxiao@sdau.edu.cn

College of Horticulture Science and Engineering; State Key Laboratory of Crop Biology, Shandong Agricultural University, Tai'an 271000, Shandong, China
}

(c) The Author(s). 2020 Open Access This article is licensed under a Creative Commons Attribution 4.0 International License, which permits use, sharing, adaptation, distribution and reproduction in any medium or format, as long as you give appropriate credit to the original author(s) and the source, provide a link to the Creative Commons licence, and indicate if changes were made. The images or other third party material in this article are included in the article's Creative Commons licence, unless indicated otherwise in a credit line to the material. If material is not included in the article's Creative Commons licence and your intended use is not permitted by statutory regulation or exceeds the permitted use, you will need to obtain permission directly from the copyright holder. To view a copy of this licence, visit http://creativecommons.org/licenses/by/4.0/ The Creative Commons Public Domain Dedication waiver (http://creativecommons.org/publicdomain/zero/1.0/) applies to the data made available in this article, unless otherwise stated in a credit line to the data. 


\section{Background}

Currently, salt stress affects over $6 \%$ of the global area (more than 800 million hectares of land), and the infected area continues to increase [1]. In plants, high salt can affect the absorption of water and nutrients and reduce photosynthesis, thereby inhibiting plant growth and causing yield loss. And at the cellular level, high salt can disturb normal metabolism and cause "physiological drought", ionic toxicity or causing complex secondary damages to proteins, nucleic acids, and other macromolecular substances [2].

Fundamentally, the salt tolerance of plants is conferred by tolerance genes. The isolation of novel tolerance genes and effective identification of salt-resistant germplasm are key strategies for improving salt tolerance in plants [3]. Recently, extensive genetic and molecular studies using loss-of-function mutants and overexpression lines have been carried out to investigate the molecular basis of salt tolerance in model plants and crop species. It has been reported that protein kinases of the mitogen activated protein kinase (MAPK), Calcineurin B-like-interacting protein kinase (CIPK) and SNF1related protein kinases family(SnRKs) families and many transcription factors (TFs), such as NAC(NAM, ATAF1, 2, and CUC2), AP2/ERF, MYB, WRKY, bZIP, were found to play important roles in environmental stress responses [4-12]. Therefore, screening for salt-tolerant genes via molecular studies is a necessity for protecting the plants against salt stress.

The structure and function of SnRK1 are similar to those of the yeast SNF1 and mammalian AMPK [13]. SnRK1 holoenzyme is a heterotrimer comprising a catalytic $\alpha$ subunit, a regulatory $\gamma$ or $\beta \gamma$ subunit, and $\beta$ subunit as a scaffold connecting $\alpha$ and $\gamma$ subunits [13]. Phosphorylation of the conserved threonine on the $\mathrm{T}$ loop of the catalytic $\alpha$ subunit is required for SNF1/ AMPK/SnRK1 to remain active [14-17].

SnRK1 is involved in sucrose and lipid metabolism in plants and can respond to stress signals under stress conditions, thereby regulating plant growth and development [18]. For example, increased SNF1 catalytic activity and phosphorylation of the conserved Thr-210 in the activation loop occur in yeast cells exposed to sodium or oxidative stress, indicating that similar to AMPK, SNF1 is activated in response to these stresses [19]. Moreover, it has been reported that SnRK1 activity is required for flooding resistance in Arabidopsis [20]. Likewise, SnRK1 is an essential kinase induced by autophagy under various stress conditions in Arabidopsis [21].

The sucrose nonfermenting-1-related protein kinases(SnRKs)family can respond to stress through regulating multiple signaling pathways via cascade amplification of the stress signal and initiate a stress response. The
SnRKs family can be further subdivided into three subfamilies-SnRK1, SnRK2, and SnRK3-according to the differences in tertiary structures [22]. In Arabidopsis, SnRK2.2/SnRK2.4/SnRK2.6 are important regulators of ABA signaling in response to abiotic stress [23], and SnRK3 indirectly participates in ABA signaling by interacting with the regulatory factors in the ABA signaling pathway, thereby enhancing stress tolerance in plants. For example, ABI2 interacts strongly with SnRK3.11/ SnRK3.13/SnRK3.15 to regulate the expression of salt stress-related genes, rendering the plants more sensitive to the stress signal and enhancing salt tolerance $[24,25]$. In Arabidopsis, SnRK1 is negatively regulated by the ABA-insensitive 1 (ABI1) and protein phosphatase 2C A group (PP2CA) [26]. Therefore, we speculate that SnRK1 is involved in abiotic stress response via ABA signaling or other pathways.

Peach (Prunus persica) is a unique plant species, probably originated from China. It contains abundant valuable genetic resources that can be used to study and improve stress tolerance in plants [27]. In this study, the full-length Prunus persica SnRK1 $\alpha$ $(P p S n R K 1 \alpha)$ sequence was obtained from Prunus persica (Linn.) Batsch. The wild-type (WT) and three transgenic tomato seedlings overexpressing $P p S n R K 1 \alpha$ (OE-1, OE-4, and OE-7) in WT were used. In order to study the possible regulatory mechanism of SnRK1 under salt stress, we compared the phenotype of the WT and transgenic tomato plants under salt stress and analyzed the physiological indexes, including the metabolic capacity of ROS, and ability to resist osmotic stress. Moreover, we sequenced the transcriptomes of the WT and three PpSnRK1 $\alpha$ overexpression lines using RNA-seq and identified approximately 1000 differentially expressed genes (DEGs). We further examined the expression levels of salt stressrelated genes that significantly correlated with SnRK1 and validated the interactions between SnRK1 and known ABA receptors, which provide evidence for a role of SnRK1 in salt stress response, signifying that SnRK1 $\alpha$ may be involved in the ABA signaling pathway or reactive oxygen (ROS) metabolism to improve tomato salt tolerance. Overall, our results demonstrate that the overexpression of $P p S n R K 1 \alpha$ is beneficial for enhancing salt tolerance in plants.

\section{Results}

PpSnRK1a overexpression lines have higher SnRK1 activity and increased salt tolerance

In order to further identify the difference between WT and $P p S n R K 1 \alpha$-overexpressing tomatoes lines (PpSnRK1 $\alpha$ oe), firstly we measured the expression levels of PpSnRK1 $\alpha$ in overexpression lines (OE-1, OE-4, and OE-7) and WT. The results are shown in Fig. 1a. PpSnRK1 $\alpha$ oe showed 


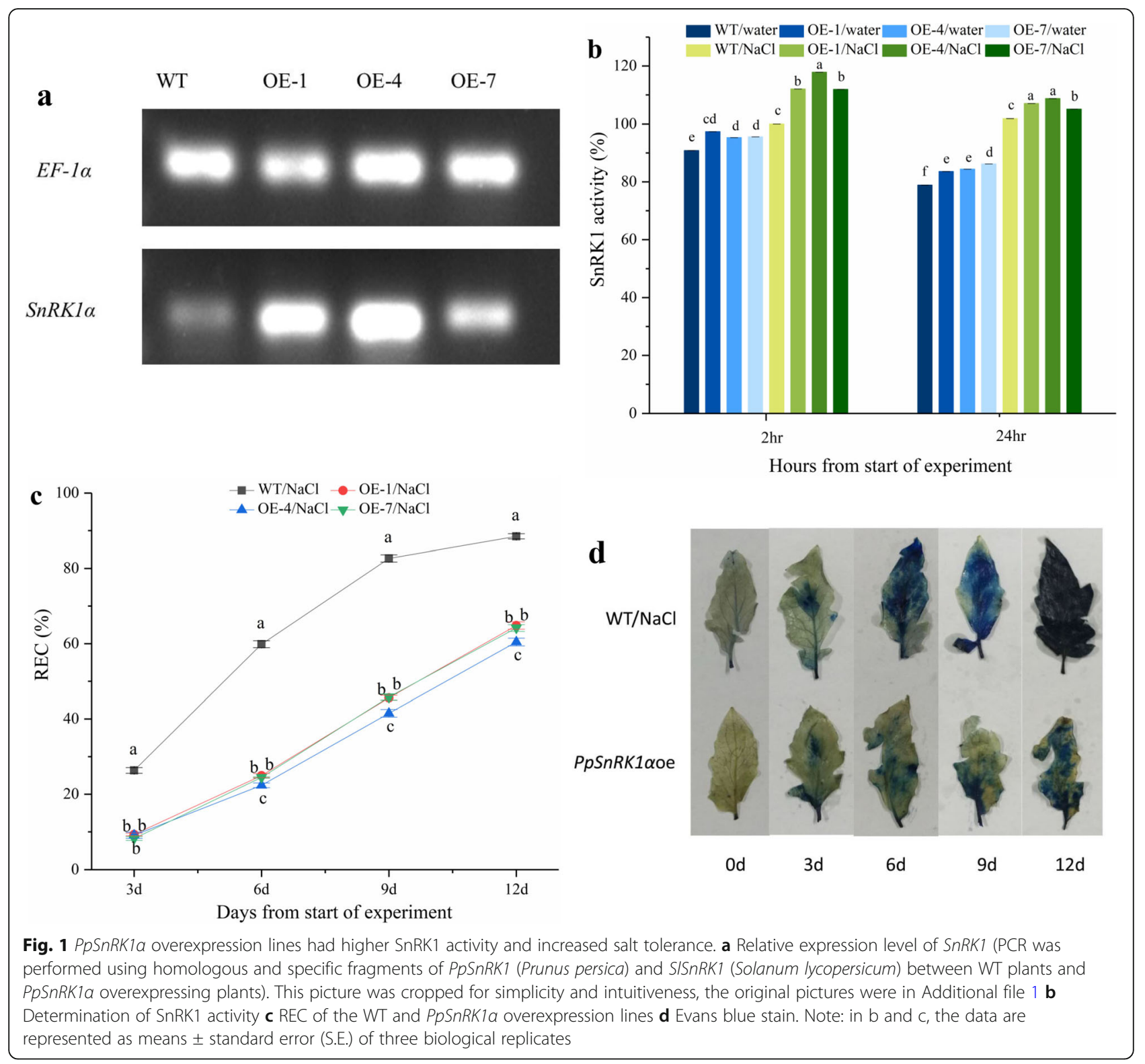

significant higher SnRK1 $\alpha$ expression levels than WT, and SnRK1 $\alpha$ expression levels were relatively higher in OE-1 and OE-4 than that of OE-7 (Fig. 1a, Additional file 1). Secondly, the SnRK1 activity of three PpSnRK1 $\alpha$ oe significantly increased following two hours of the salt treatment and was 11.9-17.8\% higher than that of the WT (Fig. 1b). However, SnRK1 activity declined after $24 \mathrm{~h}$ of high-salinity treatment both WT and PpSnRK1 $\alpha$ oe. Thirdly, the relative electronic conductance of OE-1, OE-4, OE-7, and WT were 64.84, 60.43, 64.17, and 88.49\%, respectively, after 12 days of salt treatment (Fig. 1c). In general, PpSnRK1 $\alpha$ overexpression lines exhibited a lower level of cell damage than the WT. These observations were further validated by the Evans blue staining experiment (Fig. 1d). Thus, overexpression of $P p S n R K 1 \alpha$ increased the salt tolerance of plants, and the level of improvement was proportional to the level of PpSnRK1 $\alpha$ gene expression and SnRK1 activity. Together, these results evidenced the role of PpSnRK1a overexpression in enhancing salt tolerance in tomato.

\section{PpSnRK1a regulates membrane lipid peroxidation and proline accumulation in response to salt stress}

To further determine if overexpression of PpSnRK1 $\alpha$ attenuate the degree of peroxidation of the cell membrane and accumulate proline [28] to resist salt stress, we monitored changes in the MDA content and proline content in both WT and PpSnRK1 $\alpha$ oe before and after the salt treatment. No significant difference in MDA 
content was observed between the PpSnRK1 $\alpha$ oe and WT plants under normal condition, Whereas, the MDA content in PpSnRK1 $\alpha$ oe was obviously lower than that in the WT after 12 days of high salinity treatment (Fig. 2a). On the other hand, PpSnRK1 $\alpha$ oe exhibited a more significant increase of proline content than the WT plants after 12 days of control treatment (Fig. 2b). Furthermore, proline contents accumulated to higher levels in $P p S n R K 1 \alpha$ oe than in WT under salt stress (Fig. 2b). However, the total soluble sugar content also was determined and found that there was a limited difference in soluble sugar levels between PpSnRK1 $1 \alpha$ oe and WT (Fig. 2c). Overall, these results indicate that $P p S n R K 1 \alpha$ overexpression leads to a decrease in the degree of cell membrane peroxidation and the accumulation of proline in response to salt stress.
PpSnRK1a overexpression lines exhibit reduced ROS content and higher antioxidant enzyme activities under salt stress

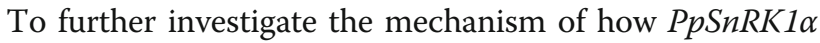
overexpression lines enhances salt tolerance in tomato, the correlation between overexpression of $P p S n R K 1 \alpha$ and reactive oxygen metabolism in plants and the differences in antioxidant enzyme activities as well as ROS content between the WT and transgenic plants were studied. After 12 days of the salt treatment, the overexpression of PpSnRK1 $\alpha$ resulted in a significant decrease $(17-26 \%)$ in the $\mathrm{O}^{2-}$ content, compared with the level in WT plants (Fig. 2a). Consistent with these observations, NBT staining revealed that the transgenic leaves were less damaged than those of the WT under salt stress (Fig. 2b).
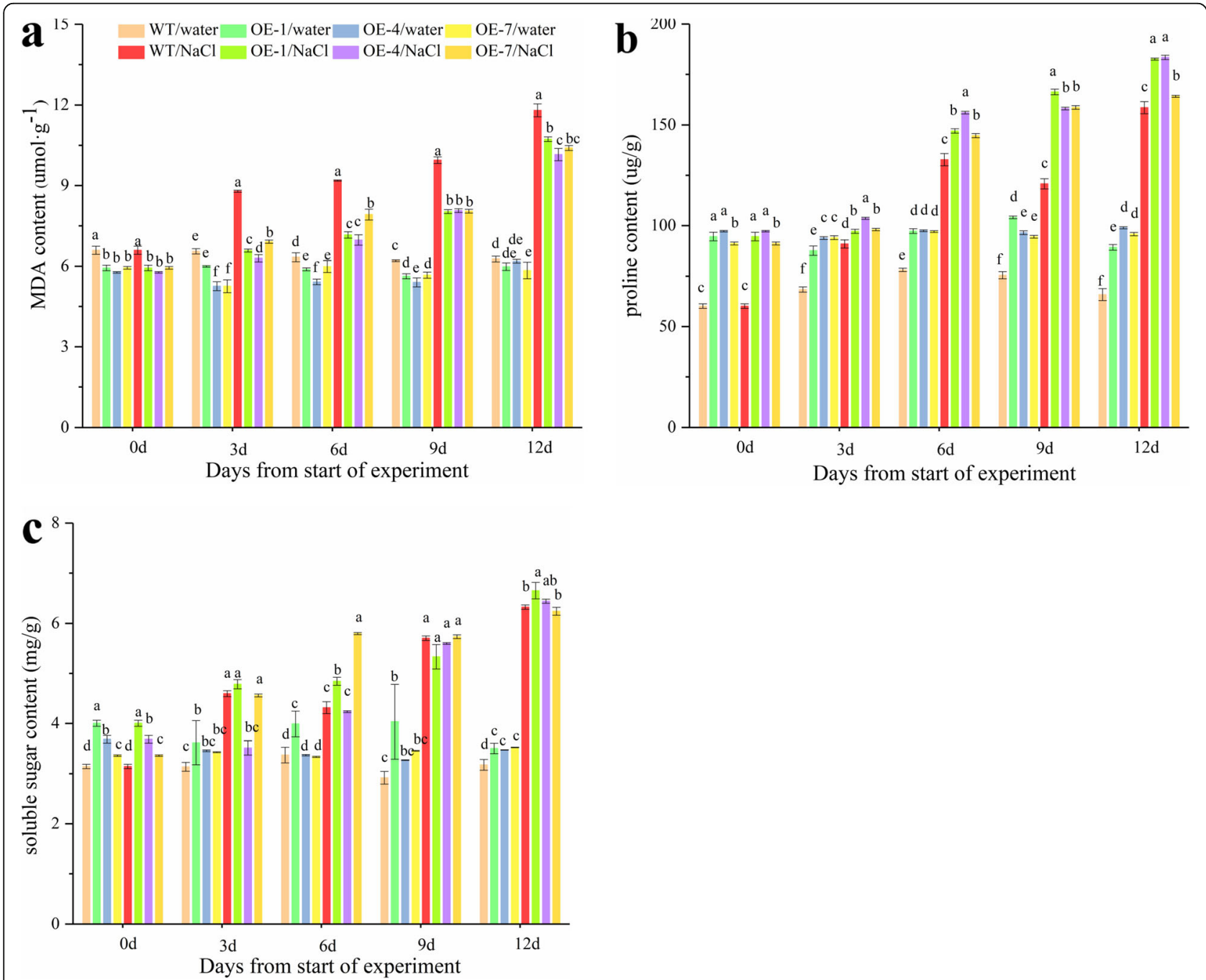

Fig. 2 MDA, soluble sugar and proline contents in the WT and PpSnRK1a overexpression lines with and without the salt treatment. a MDA content $\mathbf{b}$ proline content $\mathbf{c}$ soluble sugar content. Three biological replicates were analyzed for each sample, and the data are represented as means \pm S.E. of three technical repeats 
Under the normal growth condition, Superoxide dismutase (SOD), Catalase (CAT), Peroxisome (POD) activities of the transgenic lines were higher than those of the WT (Fig. 2c, e, f). Furthermore, the increases of SOD and POD activities in PpSnRK1 $\alpha$ overexpression lines were much more evident, $62.9-96.7 \%$ and $8.0-15.8 \%$ increases than WT after 12 days of high salinity treatment (Fig. 2e, f). What's more, PpSnRK1 1 oe exhibited higher CAT activity than the WT plants under salt stress. This result was consistent with those of the DAB staining assay, which indicated that transgenic plants have lower hydrogen peroxide content and higher CAT activity than WT (Fig. 2c, d). These results indicated that PpSnRK1 $\alpha$ may play a role in antioxidant systems to protect plants under salt stress.

Overexpression of PpSnRK1a alter the expression level of SnRK2 family genes (SnRK2.1-SnRK2.7) and antioxidase genes $(S O D, P O D$, and CAT1) in tomato

The expression levels of $S O D, P O D$, and CAT1 by Quantitative Real-Time PCR (qRT-PCR) were detected both in PpSnRK1 $\alpha$ oe and WT to further understand whether SnRK1 alters the expression of superoxide gene at the genetic level. According to Fig. 4, the transcript levels of genes encoding antioxidant enzymes (SOD, POD, and CAT) were the highest $(6.839,4.705$ and 4.421 times than WT, respectively) among others in PpSnRK1 $\alpha$ overexpression plants under high salinity condition (Fig. 4). Therefore, it can be seen that the overexpression of PpSnRK1 $1 \alpha$ increased the expression level of the antioxidant enzyme genes at the genetic or transcriptional level.

There are seven Solanum lycopersicum SnRK2 genes (SlSnRK2s) that are homologous to the Arabidopsis SnRK2.2/SnRK2.3/SnRK2.6 genes in tomato [29]. In $P p S n R K 1 \alpha$ overexpression plants, the expression level of SnRK2.1 is not significantly changed, and the expression of SlSnRK2.2, SlSnRK2.3 and SlSnRK2.5 were inhibited regardless of whether the salt stress was present or not (Fig. 4). By contrast, the expression level of SISnRK2.4 was increased in PpSnRK1 $\alpha$ oe under both conditions (Fig. 4). However, the expression level of SISnRK2.7 was inhibited under normal conditions but increased under salt stress (Fig. 4). Therefore, the overexpression of $P p \operatorname{SnRK1\alpha }$ complexly affects the expression level of SlSnRK2.

\section{Transcriptomic analysis of WT and PpSnRK1a overexpression plants}

To further evaluate the molecular mechanism of how PpSnRK1 $\alpha$ overexpression confers salt tolerance on a broader scale, the differences in gene expression profile between three PpSnRK1 $\alpha$ oe (OE-1, OE-4, and OE-7) and WT plants under normal condition were analyzed.
Three biological replicates of the WT and three transgenic lines were sequenced using the Illumina platform, and approximately 40 to 51 million high-quality reads (with Fast QC quality score > 36) were obtained for each biological replicate (Additional file 2). Of all reads obtained, 92 to $94 \%$ could be mapped to a unique chromosomal position. The expression level of each transcript in the sample was represented by Fragments Per Kilobase of transcript per Million fragments mapped (FPKM). A high linear correlation was observed among the three replicates of the same sample, suggesting small differences among replicates (Additional file 3). Differentially expressed genes (DEGs) were identified by comparing the transcriptomes of the WT and transgenic plants $[30,31]$. We identified a total of 1009 DEGs, among which 668 were upregulated and 341 were downregulated, in PpSnRK1 $1 \alpha$ overexpression plants compared with the WT (Fig. 5a). These results revealed that the expression of a large number of genes was altered upon $P p S n R K 1 \alpha$ overexpression, and these genes are either directly or indirectly regulated by SnRK1.

To test whether SnRK1 $\alpha$ overexpression relieves severe effects of salt by modulating the expression of saltresponsive genes, functional annotations of both upregulated and down-regulated genes and KEGG pathway enrichment analysis in PpSnRK1aoe were performed (Additional file 4; Fig. 5b; Additional file 5). MAPK (mitogen activated protein kinase) signaling pathwayplant, plant-pathogen interaction, and plant hormone signal transduction were main KEGG pathways that were regulated in PpSnRK1 $\alpha$ oe. Then GO terms were assigned to the DEGs. A number of genes were involved in various cellular component, molecular function, and biological processes (Fig. 5c), of which 1714 GO terms were enriched among the upregulated DEGs and 1334 GO terms among the downregulated DEGs (Additional file 6). Among the DEGs, response to stimuli, metabolic processes and biological regulation in biological process and antioxidant activity in molecular function were the obvious enriched GO terms (Fig. 5c). It is noteworthy that expression levels of 17 peroxidase genes were significantly regulated in $P p S n R K 1 \alpha$ overexpression plants, especially peroxidase 21 was upregulated 6 times and may improve the ability of transgenic plants to remove ROS under salt conditions (Additional file 4). This hypothesis was supported by the patterns of ROS accumulation in the various lines (Fig. 3).

PpSnRK1a overexpression alters the expression of several transcription factors and downstream genes, especially the transcript levels of stress-related genes Of all the DEGs, we identified 101 transcription factors (TFs) that belong to 37 families (Additional files 7 and 8). 

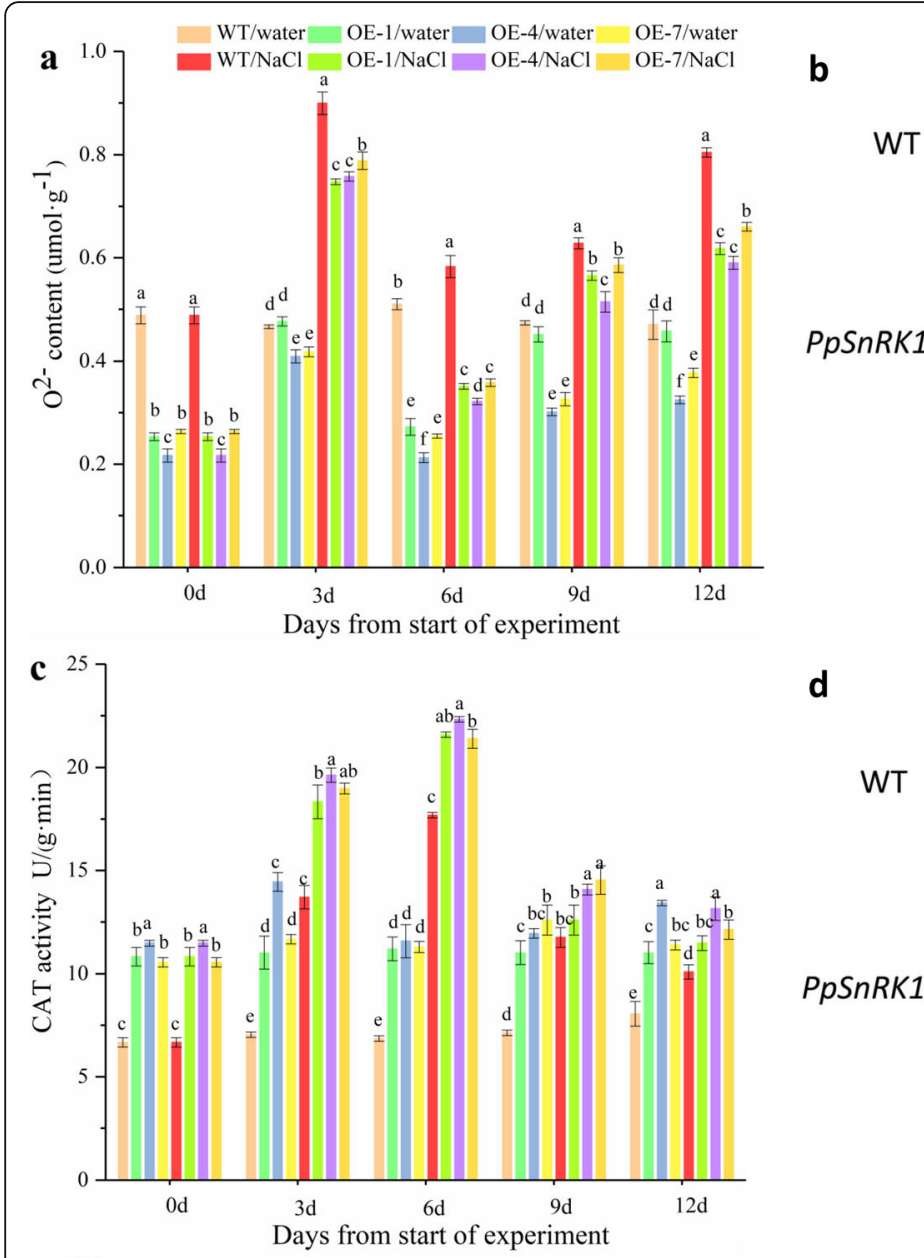

d

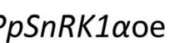

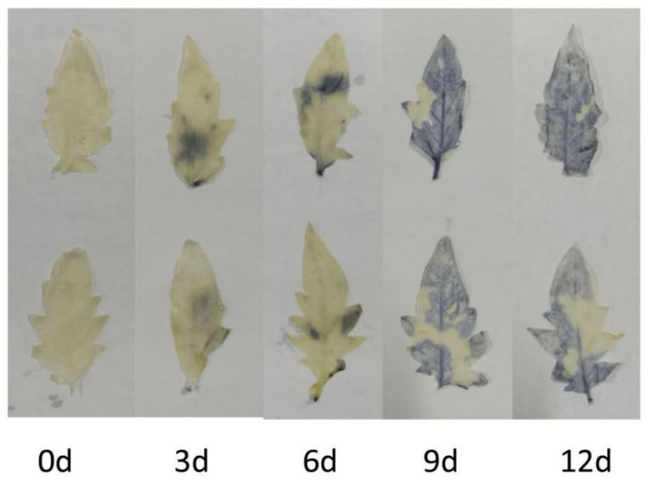

WT
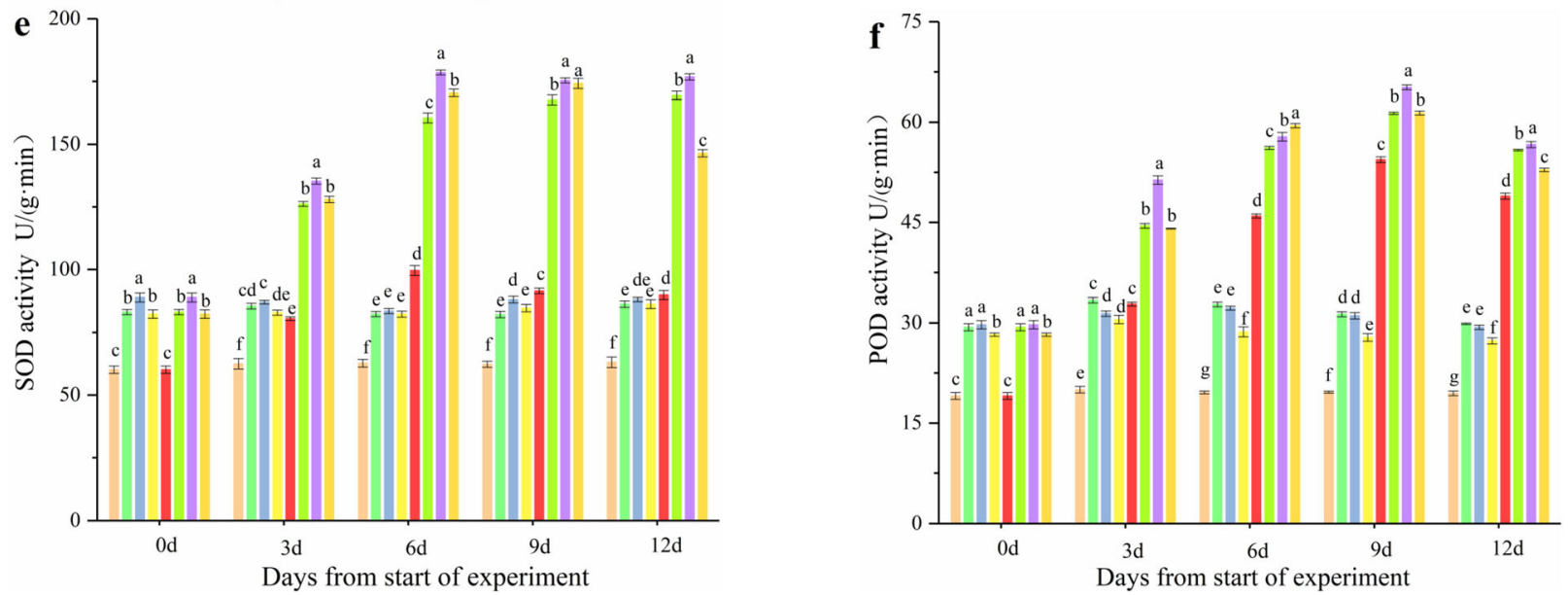

Fig. 3 Antioxidant enzyme activities and the contents of $\mathrm{O}^{2-}$ in WT and PpSnRK1a overexpression lines. a Comparison of the $\mathrm{O}^{2-}$ content between the WT and PpSnRK1a overexpression lines, $\mathbf{b}$ NBT staining for superoxide in the leaves $\mathbf{c}$ Comparison of the CAT activity between the WT and PpSnRK1a overexpression lines $\mathbf{d}$ DAB staining for $\mathrm{H}_{2} \mathrm{O}_{2}$ in the leaves $\mathbf{e}$, $\mathbf{f}$ Comparison of the SOD (e) and POD (f) activity between the WT and PpSnRK1a overexpression lines. Note: in $\mathrm{a}, \mathrm{c}$, e and $\mathrm{f}$, each sample has three biological replicates. The data are shown as means \pm S.E. of three technical repeats

Interestingly, the expression levels of TFs from the B3, GARP-G2-like, LOB, and WRKY families were upregulated, whereas those of TFs from other families, such as
GRF (growth-regulating factors) and HSF (heat shock transcription factors), were suppressed. Given that over

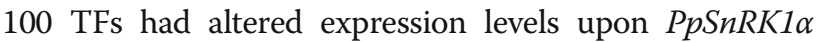


overexpression, we concluded that the TFs were directly or indirectly affected by $P p S n R K 1 \alpha$, and $P p S n R K 1 \alpha$ may regulate the expression of some genes or TFs by directly binding to their promoters.

To further investigate the role of SnRK1 in stress response, the expression levels of six stress-responsive genes between the WT and PpSnRK1 $\alpha$ oe both two conditions were compared. Of the DEGs, there were genes encoding the stress-responsive protein, including phosphatase $2 \mathrm{C}$ (solyc03g096670.3), PYL (pyrabactin resistance(PYR)like) /PYR (pyrabactin resistance)(solyc06g050500.2 and solyc01g095700.3) involved in ABA signaling pathway [32].Surprisingly, the regulated TFs also included saltrelated genes, such as NAC (No apical meristem domaincontaining protein) family, some members of which have been shown to enhance the tolerance of rice and Arabidopsis to various abiotic stresses. As shown in Fig. 6a, RNA-seq showed that SIPP2C37, a member of the abiotic stress tolerance-related $\mathrm{PP} 2 \mathrm{C}$ family, was significantly down-regulated under normal condition, and the expres-

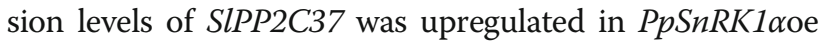
and WT under salt stress, whereas the expression level of SIPP2C37 was 1.3-fold lower than that in the WT plants (Fig. 6a, b). Furthermore, the expression levels of ABAreceptors SIPYL4 (solyc06g050500.2) and SIPYL8 (solyc01g095700.3) in PpSnRK1 $\alpha$ overexpression plants were also altered (Fig. 6c, d, e, f). For example, the SIPYL4 transcript level increased by 11 to 13-fold in PpSnRK1 ooe under both normal condition and salt stress, which was consistent with the result of RNA-seq (Fig. 6c, d); whereas PYL8 expression declined in the transgenic lines under normal condition consistent with the result of RNA-seq and slightly increased under salt stress (Fig. 6e, f). Interestingly, SINACO22 and SINACO42 were significantly upregulated higher in PpSnRK1 $\alpha$ oe than that in WT under both normal condition and salt stress, and the up-regulated expression levels of SlNACO22 and SlNACO42 in the PpSnRK1 1 oe were 359.1-fold and 52.0-fold, respectively, higher than those in the WT under salt stress (Fig. 6g, h, i, j). These data indicated that the overexpression of $\operatorname{SnRK1\alpha }$ could alter the expression of stress-related genes, and had the potential for improving the salt tolerance of tomato plants.

\section{Discussion}

When plant responses to salt stress, some protein kinases are activated, which then regulates a large number of defense-related genes, and these changes are conducive to the establishment of defense mechanisms in plants. The response of plants to salt stress is closely related to the SnRKs family, which can be through the SOS (Salt Overly Sensitive) signaling pathway and ABA signal transduction [2]. Although it is unclear how plants respond specifically to stress, there is ample evidence that SnRKs can participate in resistance to salt stress in many species $[18,33,34]$.

As a key component of the cell signaling network, SnRK1 can interact with sugar molecules of different forms and energy states, regulate the activities of other kinases, and interact with TFs to maintain energy balance within the cell under stress conditions [18]. To our

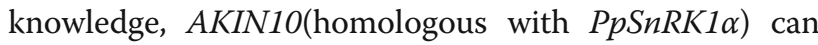
negatively regulate the accumulation of AtMYC2 protein via AKIN10 kinase activity-dependent protein modification, thereby responding to abiotic stress [35]. SnRK1 also controls $\mathrm{Na}^{+}$flux and maintains $\mathrm{Na} / \mathrm{K}$ homeostasis under high salinity stress [36]. Moreover, SnRK1 can respond to high or low glucose levels in the dark as well as under hypoxia and saline conditions, and regulate energy metabolism in plants under stress [37]. In this study, we identified PpSnRK1 $\alpha$ and showed that ectopically expressing it in tomato enhanced salt tolerance phenotypes (Fig. 1). Consistent with previous studies, overexpressing PpSnRK1 $\alpha$ lines had higher cell viability and grew better under salt stress, which was accompanied by higher $P p S n R K 1 \alpha$ expression level and SnRK1 activity (Fig. 1), indicating that $S n R K 1$ is a positive regulator of salt tolerance in tomato. Therefore, PpSnRK1 $\alpha$ may have potential additional mechanisms in addition to its role in salt tolerance.

Accumulation of reactive oxygen species is one of the main responses of plants to salt stress. Excessive ROS will destroy biological macromolecules and produce toxic effects on cells. Several antioxidant enzymes in plants, such as antioxidant dismutase, peroxidase, and catalase, play critical roles in the process of scavenging ROS [38]. It was reported that ROS could also be used as a signaling molecule to mediate plant responses to different stresses [39, 40]. Some protein kinases in the cell can sense the stimulation of exogenous ROS or the production of ROS, and respond to stress through a series of phosphorylation and dephosphorylation signals. For example, $\mathrm{NADPH}$ oxidase can rapidly increase the intracellular ROS level, and the stimulus signal quickly enters the nucleus from the plasma membrane of the cell [41]. Szymańska, P. Katarzyna et al. found that two SNF1releted protein kinases 2 (SnRK2), SnRK2.4 and SnRK2.10, could regulate ROS homeostasis and respond to salinity in Arabidopsis [42]. In our investigation, overexpressing $P p S n R K 1 \alpha$ lines had increased ROS scavenging capacity, low degree of cell membrane damage, and remarkably higher expression of antioxidant enzyme genes under salt stress, which could partially explain the mechanism responsible for the improved salt tolerance of the transgenic plants

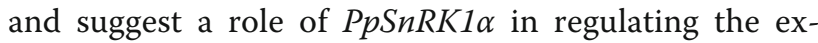
pression of antioxidase genes (Figs. 2, 3, 4, and 7). In 


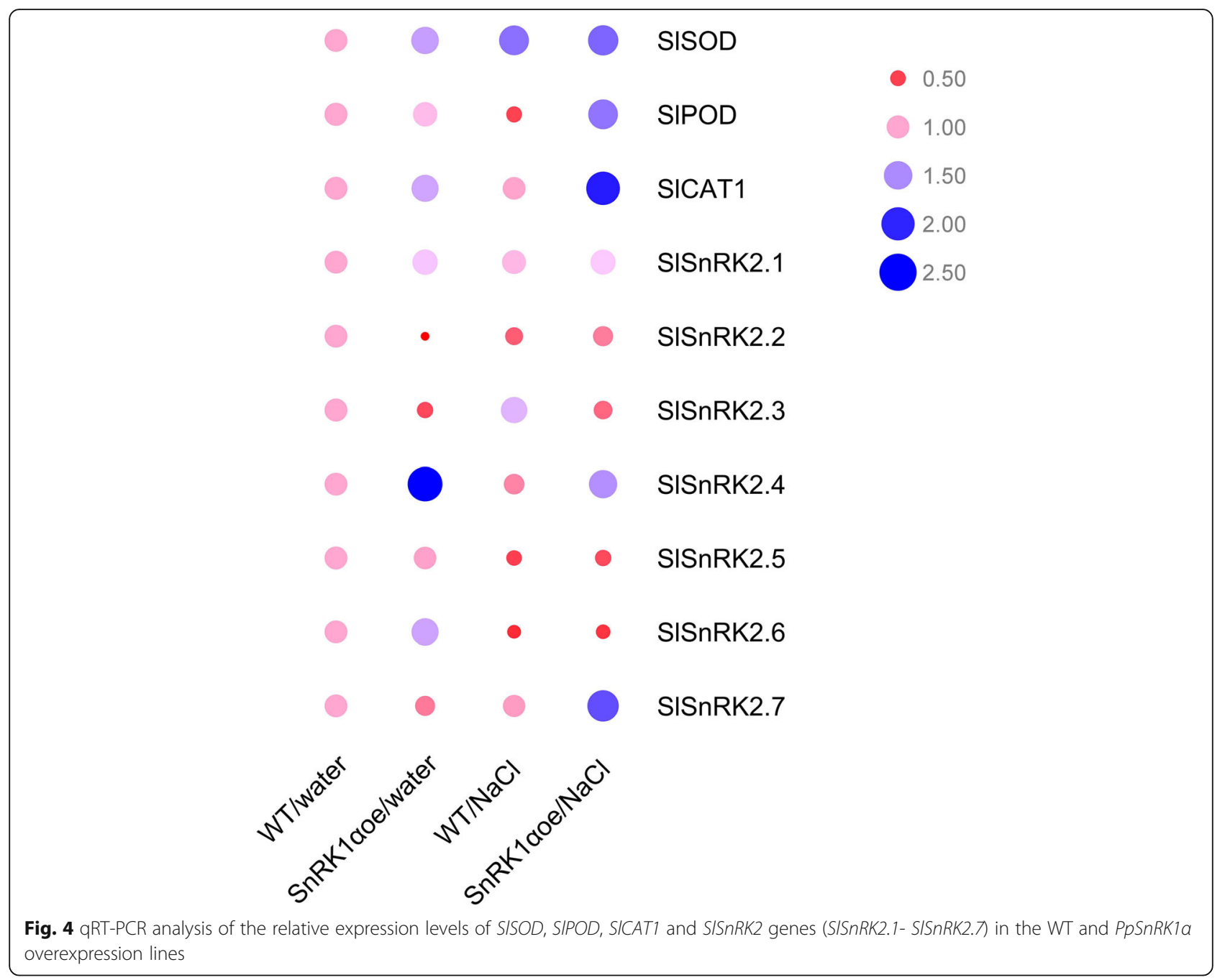

general, proline is considered to be one of the osmotic regulators in wheat to resist salt stress, and the accumulation of proline help maintain the integrity and function of the cellular vacuolar membrane, thereby enhancing its ability to resist salt damage $[43,44]$. It is apparent that $P p S n R K 1 \alpha$ helps to maintain the integrity and function of cell membranes, thus enhancing the plant's ability to resist salt damage (Fig. 2). Therefore, we think that these may have contributed, at least partially, to the observed salt tolerance.

The SnRKs represent a large family that can be further divided into the SnRK1, SnRK2, and SnRK3 subfamilies. Many members of the SnRKs family have been shown to play key roles in abiotic stress responses. AREB1/ABF2, AREB2/ABF4, and ABF3 are activated by SnRK2.2, SnRK2.6 andSnRK2.3 in ABA signaling in response to osmotic stress during vegetative growth [45]. SnRK2.2 and the closely related SnRK2.3 are known to play roles in $\mathrm{ABA}$ inhibition of seed germination in Arabidopsis [46]. Additionally, SnRK2.6 can interact with ABAR/
CHLH and response to abscisic acid in guard cell signaling [23], The results showed that when plants were

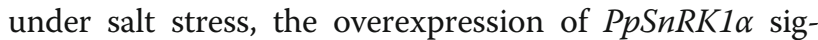
nificantly affected the expression levels of seven $S n R K 2$ family genes in tomato (Fig. 4). Moreover, the research about the SnRKs family revealed that there are also interactions between different genes in the family. For example, in Arabidopsis, SnRK3.15 can interact with SnRK1.1 and SnRK1.2, regulate the activity of them and participate in sugar metabolism [47]. Therefore, the direct or indirect interaction between members of different subfamilies of the plant SnRKs strengthens the plant's ability to respond to stress.

Abscisic acid is a key player in regulating abiotic stress response in plants. A previous study has evidenced the interaction between SnRK1.1 and ABA signaling-the crossing of an SnRK1.1 overexpression Arabidopsis line with the aba2 mutant, which was impaired in both ABA signaling transduction and sugar signaling pathway, led to glucose hyposensitivity [48]. In addition, defective 
kernel 33 (DEK33) is involved in the ABA synthesis process and can interact with SnRK1, suggesting that their interaction may regulate the stability of DEK33 and thus the ABA signal [49]. It was reported that inhibition of $S n R K 1$ expression in peas resulted in pleiotropic maturation defects similar to those of the ABA insensitive mutants and SnRK1 can respond to ABA treatment in wheat roots, the level of which drops sharply, but the amount of phosphorylated (active) SnRK1 remains constant $[50,51]$. In our study, we found that the expression of SIPP2C37 (a member of PP2C family) [26], SlPYL4, SIPYL8 (an ABA receptor in ABA signaling) and SnRK2 family genes were significantly regulated upon PpSnRK1a overexpression under the normal growth condition as revealed by the RNA-seq data (Figs. 5, 6 and 7). MAPK (mitogen activated protein kinase) cascades have been shown to be implicated in ABA signaling and abiotic stress as well [32]. The RNA-seq results showed that the MAPK pathway was significantly regulated in the PpSnRK1 $\alpha$ overexpression lines (Fig. 5, Additional file 4). These genes have been shown to be involved in the ABA signaling pathway [5]. According to these results, we presumed that $S n R K 1 \alpha$ may be involved in the ABA signal transduction pathway. Further verification is needed to illustrate the regulatory mechanism

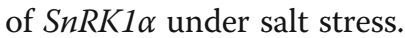

NAC proteins, as one of the largest family of transcription factors unique to plants, are known to possess diverse roles in plant response to environmental stress [52]. It was reported that $A N A C 036$ (NAC transcription factor family member in Arabidopsis) involved in inflorescence and leaf morphogenesis in Arabidopsis [53]. And Hong $\mathrm{Y}$ et al. found that ONAC22 (NAC transcription factor family member in rice) played a positive role in drought and salt stress tolerance through modulating an ABA-mediated pathway [54]. Our investigation

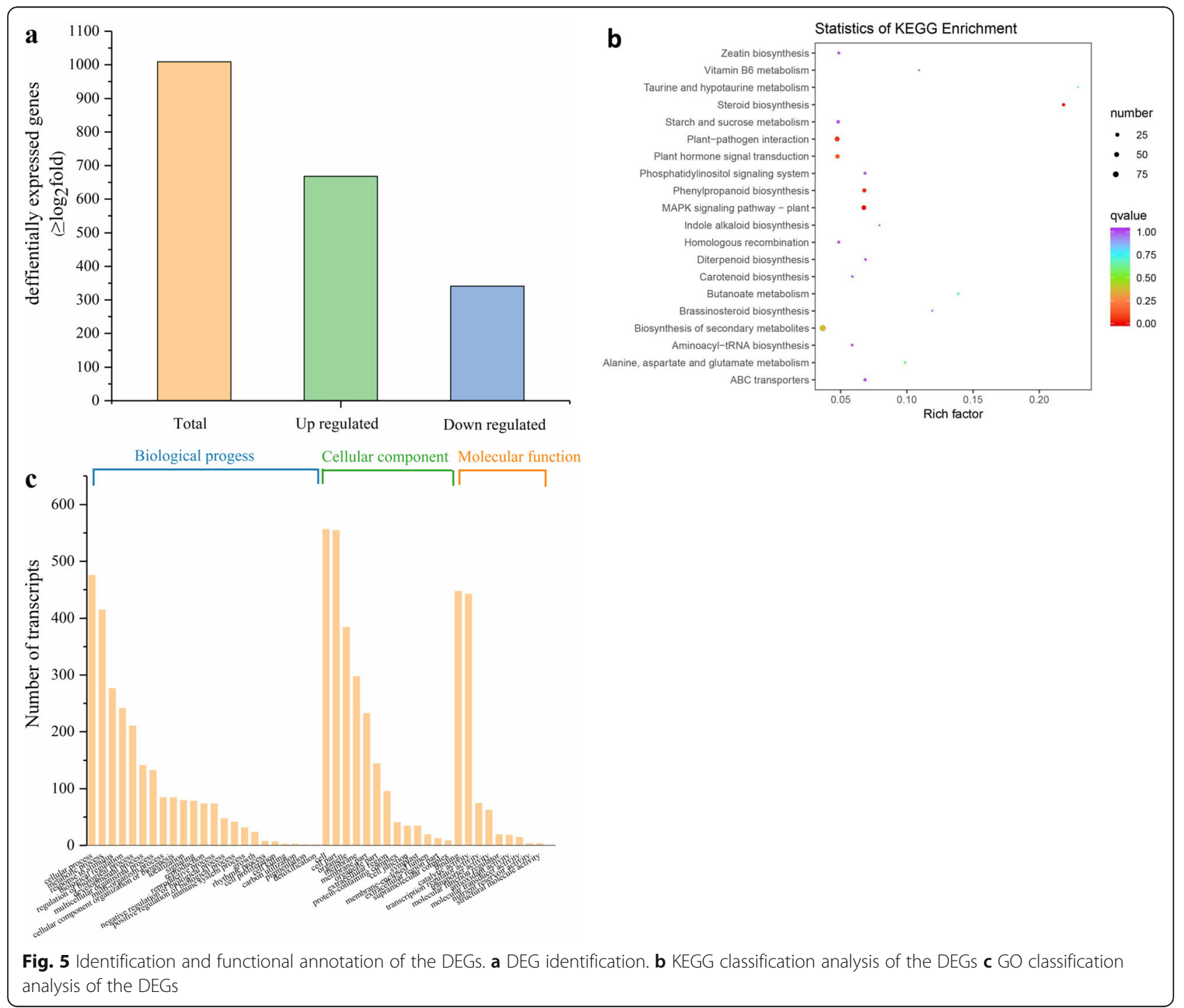




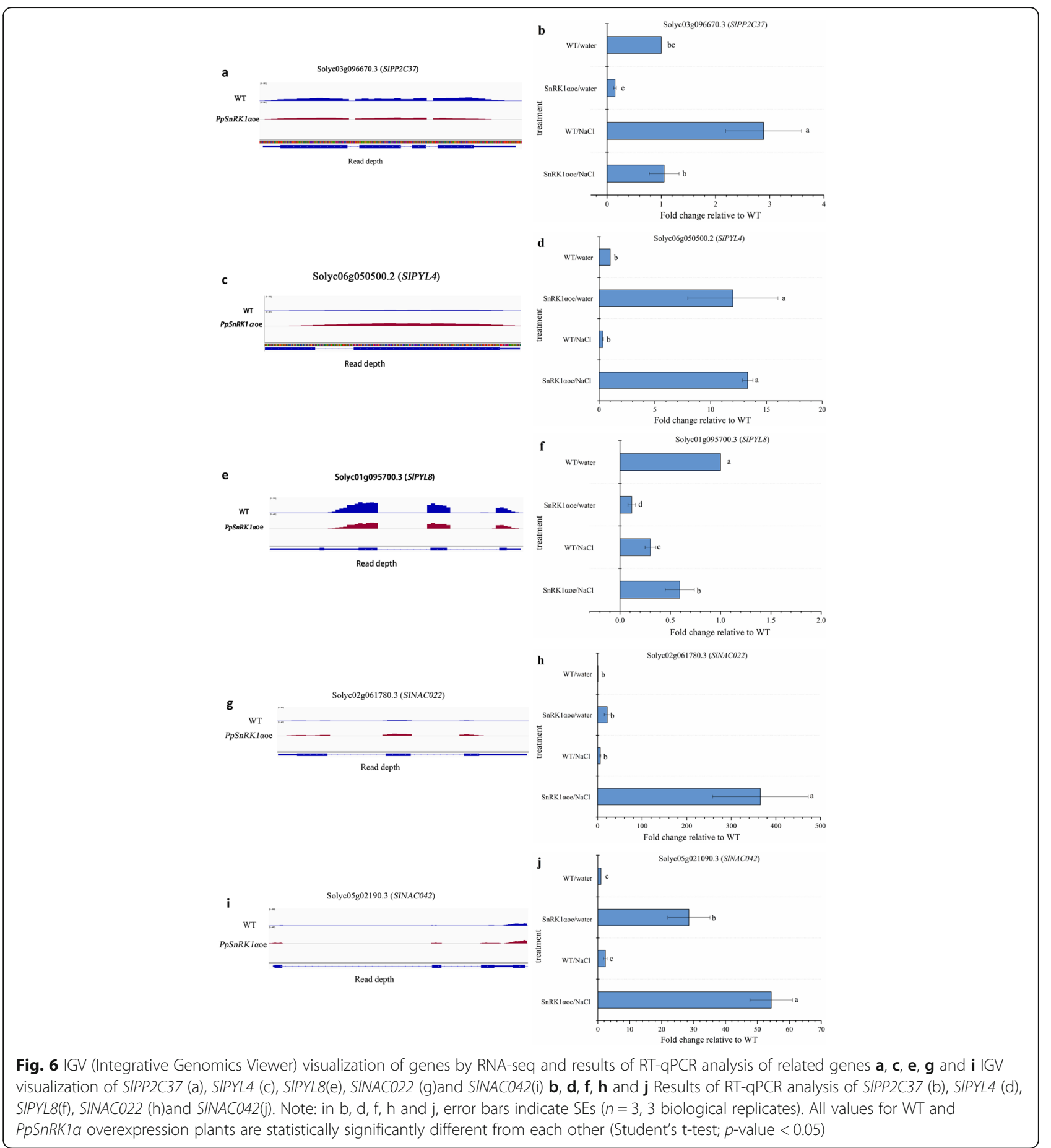

showed that SINACO22, which has high homology with $A N A C 036$ and $O N A C 22$, was significantly regulated in $P p S n R K 1 \alpha$ overexpressing plants (Fig. 6). Overexpression of JUNGBRUNNEN1 (JUB1) can strongly delay senescence and enhanced tolerance to various abiotic stresses in Arabidopsis [55, 56]. Interestingly, the expression level of SINAC42, which has high homology with JUB1, is also significantly higher than that in WT plants (Fig. 6).
Here we hypothesize that the up-regulation of SINACO22 and SINAC42 in PpSnRK1 $\alpha$ overexpressing leaves enhances plant salt tolerance in response to ABA signals. More work is required to further determine if SnRK1 and NAC022/ NAC42 interact directly to improve salt tolerance.

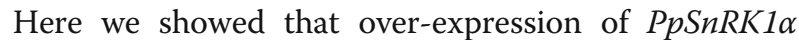
improved the salt tolerance of plants. These observations 


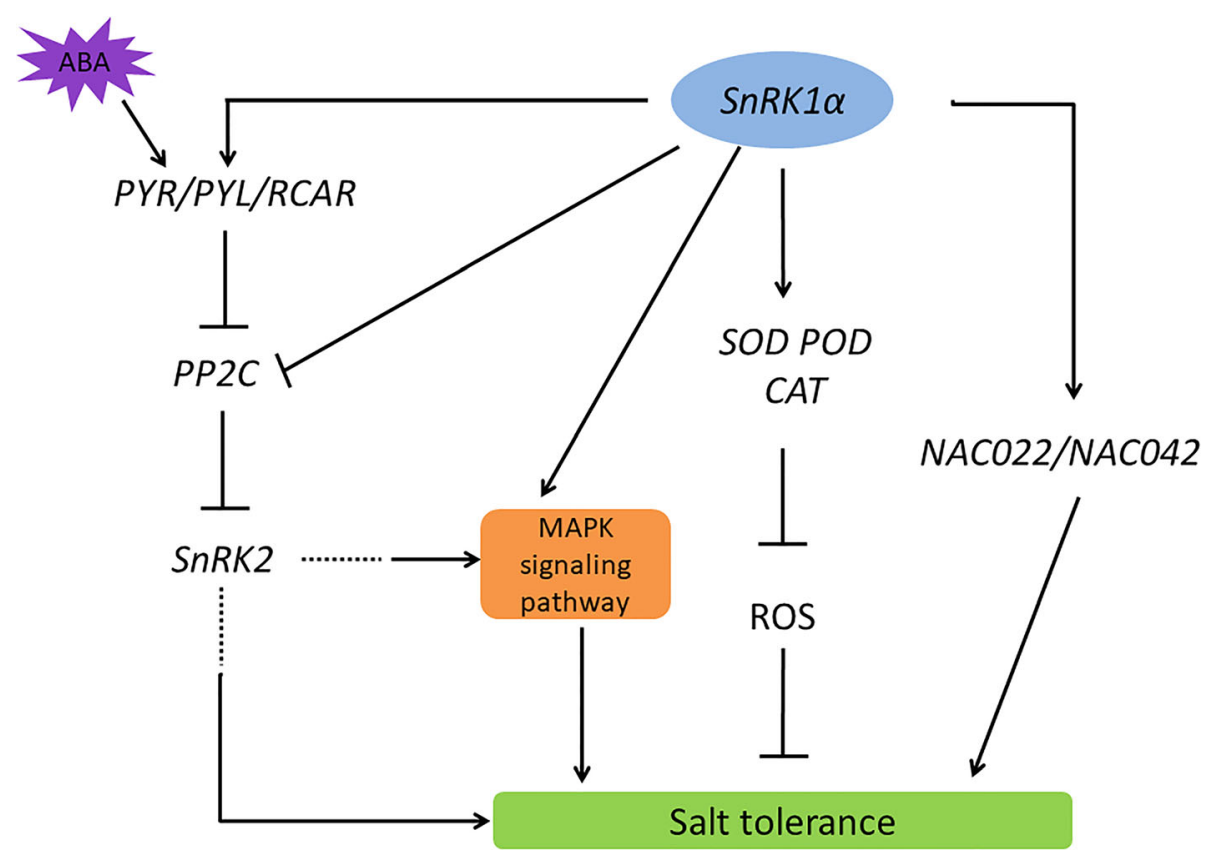

Fig. 7 The working model of PpSnRK1a regulating salt tolerance through the ABA signal transduction pathway and ROS mechanism. In the current working model, PPSnRK1a-overexpression improves the transcription levels of SOD, POD, and CAT1 as well as SOD, POD and CAT activity, reduces ROS production in plant cells. PpSnRK1a is involved in the ABA signaling pathway by altering the transcription levels of ABA receptors (SIPYL4, SIPYL8), PP2C (SIPP2C37) and SISnRK2s, and thereby leads to an increase in tolerance to salt stress in tomato. Additionally, PpSnRK1aoverexpression increases the expression of SINACO22, which has been proved to participate in ABA signaling transcription

indicated that stress resistance could be affected by the regulation of the PpSnRK1 $\alpha$ gene. Further molecular and genetic analysis will increase our understanding of the function of PpSnRK1 $\alpha$ in plant stress resistance and provide strategies for breeding transgenic crop varieties with high-resistance.

\section{Conclusions}

In summary, the results of this study indicate that SnRK1 functions as a key kinase for stress response and $P p S n R K 1 \alpha$ overexpression can significantly improve salt tolerance via regulating ROS metabolism or possibly ABA-mediated pathways. What's more, SnRK1 may direct or indirect interacts with the SnRK2 family. The mechanism by which SnRK1 responds to salt stress is complex, and whether SnRK1 directly regulates NACO22 and NAC042 expression or indirectly controls their transcription by bind to intermediate receptors warrants further investigations. Generating snrk1 loss-of-function mutants might be necessary to further understand the molecular mechanisms underlying the function of SnRK1 in salt stress response and tolerance.

\section{Methods}

Vector construction and tomato transformation

In this study, the WT tomato (Solanum lycopersicum Mill. cv. Zhongshu 6) were kindly provided by Qing-wei Meng [57]. Then we obtained over-expressing
$P p S n R K 1 \alpha$ tomatoes by vector construction and tomato transformation, and selected three lines (OE-1, OE-4, and OE-7) for further research.

Based on the coding sequence of PpSnRK1 (https:// www.ncbi.nlm.nih.gov/nuccore/XM_007215174.2), two primers were designed for the amplification (forward primer: 5' - GCTCTAGAATGGATGGATCGGTTG-3'; reverse primer: $5^{\prime}$ - GCGTCGACTTAAAGGACCCG $-3{ }^{\prime}$ ). Then, the PpSnRK1 $\alpha$ coding sequence was inserted in the sense orientation into the binary vector pBI121 downstream of the $35 \mathrm{~S}$ promoter of the cauliflower mosaic virus (35S: PpSnRK1 $\alpha$ ). The construct was introduced into the Agrobacterium tumefaciens strain LBA4404 for tomato (Solanum lycopersicum Mill. cv. Zhongshu 6) transformation using the leaf disc method [58].

T2 transgenic plants overexpressing PpSnRK1 $\alpha$ were identified by the T5 direct PCR kit (TSINGKE, TSE011) using primers $P p S n R K 1 \alpha$-DF (5'-GTGTGGAGTTGCGG AGTCAT-3') and PpSnRK1 $\alpha$-DR (5'-ACGAGGAAGA TGAGCCTGGA-3'). The PCR-positive tomato plants were transplanted into pots with soil and grown under natural conditions. Next, we selected three transgenic tomato lines (OE-1, OE-4, and OE-7) for further research.

\section{Plant materials and treatments}

The WT and transgenic tomato seeds were cultured for 4 weeks in the greenhouse at $25 \pm 3{ }^{\circ} \mathrm{C}$ under a $16 \mathrm{~h}$ light 
$18 \mathrm{~h}$ dark cycle. After 1 month, the WT and the three transgenic lines, OE1, OE4, and OE7, were treated by either water (the control treatment) or $100 \mathrm{mmol} \cdot \mathrm{L}^{-1}$ sodium chloride $(\mathrm{NaCl})$. Each treatment contained three groups with ten seedlings per group. Salt stress was introduced by watering the plants with $1000 \mathrm{~mL} 100$ mmol.L-1 $\mathrm{NaCl}$ solution until saturation every 3 days. The leaves samples were collected at days $0,3,6,9$ and 12 after the treatment. SnRK1 enzyme activity was measured two and $24 \mathrm{~h}$ following the $\mathrm{NaCl}$ treatment on day one.

\section{RT-PCR}

Total RNA was extracted from tomato leaves using Ultrapure RNA Kit (CWBIO, China, CW0581M) and reverse transcribed into cDNA using PrimeScript ${ }^{\text {ti }}$ II 1 st Strand cDNA Synthesis Kit (Takara, 6210A). The volume of each cDNA pool was adjusted to give the same PCR signal strength for EF1 $\alpha$ after 32 cycles. PpSnRK1 and SISnRK1 homologous segments were selected to de-

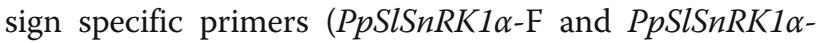
R) for PCR identification (Additional file 9). PCR was carried out using the Premix Taq (Takara, R004A).

\section{SnRK1 activity determination}

One gram of each fresh plant sample harvested at two and $24 \mathrm{~h}$ after the salt treatment, was ground in $1 \mathrm{~mL}$ of cold extraction buffer containing $100 \mathrm{mM}$ Tricine$\mathrm{NaOH}$ (pH 8.0), $25 \mathrm{mM} \mathrm{NaF}, 5 \mathrm{mM}$ dithiothreitol, $2 \mathrm{mM}$ tetrasodium pyrophosphate, $0.5 \mathrm{mM}$ ethylene diamine tetra acetic acid, $0.5 \mathrm{mM}$ ethylene glycol tetra acetic acid, $1 \mathrm{mM}$ benzamidine, $1 \mathrm{mM}$ phenylmethylsulfonyl fluoride, $1 \mathrm{mM}$ protease inhibitor cocktail (Sigma P9599), phosphatase inhibitors (PhosStop; Roche), and insoluble polyvinylpyrrolidone to the solution with a final concentration of $2 \%(\mathrm{w} / \mathrm{v})$. The homogenate was transferred to two cold microfuge tubes and centrifuged at $12,000 \times g$ for $5 \mathrm{~min}$ at $4{ }^{\circ} \mathrm{C}$. The supernatant $(750 \mathrm{uL})$ was desalted on a $2.5 \mathrm{~mL}$ centrifuge column (Sephadex G-25 medium columns; GE Healthcare) that was pre-equilibrated. SnRKl activity was determined by the Universal Kinase Activity Kit (R\&D Systems, Minneapolis, MN, United States, EA004) by using AMARA polypeptide as the substrate [59].

\section{Determination of antioxidant capacity and the content of proline}

Proline content was evaluated by the acid ninhydrin method [60]. The $\mathrm{O}^{2-}$ content was quantified by the hydroxylamine oxidation method [61]. 3,3'-diaminobenzidine (DAB) staining and nitroblue tetrazolium (NBT) staining were performed to detect $\mathrm{H}_{2} \mathrm{O}_{2}$ and $\mathrm{O}^{2-}$ levels in tomato leaves. Specifically, tomato leaves were first immersed in a $0.5 \mathrm{mg} / \mathrm{mL}$ DAB staining solution $(\mathrm{pH}=$
3.8) or $0.5 \mathrm{mg} / \mathrm{ml} \mathrm{NBT}$ solution, and then placed in a dark, high-humidity plastic box for about $24 \mathrm{~h}$. Next, the staining solutions were decanted and the leaf samples were incubated with an ethanol/lactic acid/glycerol solution (3:1:1, v:v:v) in a boiling water bath for 5 min [62]. The SOD activity was determined by the photochemical reduction method using nitroblue tetrazolium [63]. The CAT activity was determined by the ultraviolet absorption method and change in the optical density at $240 \mathrm{~nm}$ (OD240) of 0.1 within 1 min was defined as one unit of enzyme activity [64]. POD activity was measured by the guaiacol method at OD470, and one unit enzyme activity was defined as 0.1 decease in OD470 per minute [65]. SOD, POD, and CAT activities are shown as $\mathrm{U} \cdot \mathrm{min}^{-1} \cdot \mathrm{g}^{-}$ ${ }^{1}$ (FW).

The degree of damage to the cells was determined by measuring the relative conductivity of tomato leaves. Ten leaf disks $(0.8 \mathrm{~cm}$ in diameter $)$ from the WT or transgenic lines were submerged in $20 \mathrm{~mL}$ distilled water, vacuumed for $30 \mathrm{~min}$, shaken for $3 \mathrm{~h}$ at room temperature, and measured for the initial electric conductivity (S1). Subsequently, these leaf samples were boiled for $30 \mathrm{~min}$ and cooled down to room temperature to measure the final electric conductivity (S2). The electric conductivity of pure distilled water was used as the blank (S0). The relative electronic conductance (REC) was calculated as $\mathrm{REC}=\frac{\mathrm{S} 1-\mathrm{S} 0}{\mathrm{~S} 2-\mathrm{S} 0} * 100 . \mathrm{MDA}$ content was determined by the thiobarbituric acid method [66]. Tomato leaves were stained with the Evans blue staining solution to assess cell viability [67].

\section{RNA-seq}

Leaves of WT and three PpSnRK1 $\alpha$ overexpressing lines of tomato grown under normal conditions for 2 months were collected. RNA isolation, quality control, library construction, and Illumina Hiseq sequencing were performed by the Wuhan Metware Biotechnology co., LTD.

\section{Bioinformatic analysis Quality control}

Raw reads were removed low-quality reads and reads containing adapters and poly- $\mathrm{N}$ from the raw data, then filtered and examined for the sequencing error rate and GC content. The resulting clean, high-quality reads were used for subsequent analyses.

\section{Mapping and differentially expressed gene (DEG) identification}

The sequences were mapped to the tomato genome using HISAT2. The FPKM and count value were calculated using feature Counts. DESeq2 was used to analyze the differences between different samples and treatment groups $[30,31]$. Then Multiple hypothesis test correction was 
performed using the Benjamin-Hochberg method at a significance level of $P<0.05 ; \mid \log 2$ fold change $\mid \geq 1$ was used to identify differentially expressed genes (DEGs) between the two libraries (PpSnRK1 1 oe and WT). The Gene Ontology (GO) and Kyoto encyclopedia of Genes and Genomes (KEGG) enrichment analyses of the DEGs were performed using $\mathrm{R}$ based on the hypergeometric distribution.

\section{qRT-PCR}

Quantitative Real-Time PCR (qRT-PCR) was carried out using the UltraSYBR mixture (CWBIO, China, CW2601M). The $25 \mu \mathrm{L}$ reaction mixture contained $0.5 \mu \mathrm{L}$ of forward primer $(10 \mu \mathrm{M}), 0.5 \mu \mathrm{L}$ reverse primer $(10 \mu \mathrm{M}), 5 \mathrm{ng} \mathrm{cDNA}$ template, $12.5 \mu \mathrm{L} 2 \times$ UltraSYBR mixture, and $9.5 \mu \mathrm{L}$ water. The conditions used for qRT-PCR were as follows: $95^{\circ} \mathrm{C}$ for $10 \mathrm{~min}$, followed by 40 cycles of $95^{\circ} \mathrm{C}$ for $10 \mathrm{~s}, 60^{\circ} \mathrm{C}$ for $30 \mathrm{~s}$, and $72{ }^{\circ} \mathrm{C}$ for $32 \mathrm{~s}$. The calculation method for qRTPCR is $2^{-\Delta \Delta C t}$ with the $E F-1 \alpha$ gene as the endogenous control. Each sample had three independent biological replicates. Refer to Additional file 8 Table S6 for primers used for RT-PCR.

\section{Statistical analysis}

Statistical analysis was performed using Microsoft Office Excel 2010 and images were processed with Microsoft PowerPoint 2010. Comparison of the mean values was performed by the Duncan multi-range test in SPSS 20.0. $\mathrm{P}<0.05$ denotes significant differences.

\section{Supplementary information}

Supplementary information accompanies this paper at https://doi.org/10. 1186/s12870-020-02342-2.

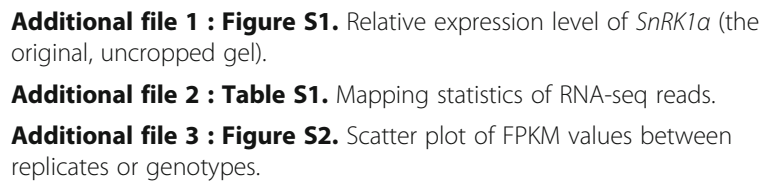

Additional file 4 : Table S2. List of DEGs and their expression levels in WT and PpSnRK1a overexpression lines.

Additional file $\mathbf{5}$ : Table S3. KEGG enrichment analysis.

Additional file $\mathbf{6}$ : Table $\mathbf{S 4}$. $\mathrm{GO}$ enrichment analysis.

Additional file $\mathbf{7}$ : Figure S3. 101 TFs of 37 families regulated by PpSnRK1a.

Additional file 8 : Table S5. List of TFs and their expression levels in WT and PpSnRK1a overexpression lines.

Additional file $\mathbf{9}$ : Table $\mathbf{5 6}$. primers used in this study.

\section{Abbreviations}

WT: Wild-type; PpSnRK1a: SNF-related Kinase 1a subunit (Prunus persica); PpSnRK1aoe: PpSnRK1a-overexpressing tomatoes lines; ROS: Reactive oxygen species; qRT-PCR: quantitative real-time PCR; ABA: Abscisic acid; REC: Relative electronic conductance; DEGs: Differentially expressed genes;

FPKM: Fragments per kilobase of exon per million fragments mapped; GO: Gene ontology consortium; KEGG: Kyoto encyclopedia of genes and genomes; MAPK: Mitogen activated protein kinase; CIPK: Calcineurin B-like- interacting protein kinase; ABI1: ABA-insensitive 1; PP2CA: Protein phosphatase 2C A group; TFs: Transcription factors; GRF: Growth-regulating factors; HSF: Heat shock transcription factors; PYL/PYR: Pyrabactin resistance(PYR)like/ pyrabactin resistance; NAC: NAM, ATAF1,2, and CUC2; SOS: Salt overly sensitive

\section{Acknowledgments}

We would like to thank the National Natural Science Foundation of China, the National Modern Agroindustry Technology Research System Fund and "Double Tops" Program Foundation of Shandong Province for their financial support.

Authors' contributions

FP and YX conceived and designed the experiments. WRW and JHL performed the experiments. GFW, MXS, and YSX contributed reagents, materials, and analysis tools. WRW and YSX wrote the article. All authors read and approved the final manuscript.

\section{Funding}

This work was supported by The National Modern Agroindustry Technology Research System Fund (No.CARS-30-2-02), National Natural Science Foundation of China (No. 31672099 and 31801812), and "Double Tops" Program Foundation of Shandong Province (SYL2017YSTD10). The funders did not play a role in the experimental design of the study, results analysis or writing of the manuscript, but did provide financial support for the manuscript.

\section{Availability of data and materials}

The datasets supporting the conclusions of this article are included within the article and its additional files. The coding sequence of PpSnRK1a sequence is available at NCBI (https://www.ncbi.nlm.nih.gov/nuccore/XM_ $007215174.2)$.

Ethics approval and consent to participate Not applicable.

\section{Consent for publication}

Not applicable.

\section{Competing interests}

The authors declare that they have no competing interests.

Received: 18 October 2019 Accepted: 16 March 2020

Published online: 26 March 2020

References

1. Yang Y, Guo Y. Elucidating the molecular mechanisms mediating plant saltstress responses. New Phytol. 2018;217(2):523-39.

2. Zhu J-K. Abiotic stress signaling and responses in plants. Cell. 2016;167(2): 313-24.

3. Munns R. Genes and salt tolerance: bringing them together. New Phytol. 2005;167(3):645-63.

4. de Zelicourt A, Colcombet J, Hirt H. The role of MAPK modules and ABA during abiotic stress signaling. Trends Plant Sci. 2016;21(8):677-85.

5. Zhu J-K. Salt and drought stress signal transduction in plants. Annu Rev Plant Biol. 2002;53:247-73.

6. Fujii $\mathrm{H}$, Zhu J-K. Osmotic stress signaling via protein kinases. Cell Mol Life Sci. 2012;69(19):3165-73.

7. Manik SMN, Shi S, Mao J, Dong L, Su Y, Wang Q, Liu H. The calcium sensor CBL-CIPK is involved in Plant's response to abiotic stresses. Int J Genomics. 2015:2015:493191.

8. Banerjee A, Roychoudhury A. WRKY proteins: signaling and regulation of expression during abiotic stress responses. TheScientificWorldJournal. 2015; 2015:807560

9. Mizoi J, Shinozaki K, Yamaguchi-Shinozaki K. AP2/ERF family transcription factors in plant abiotic stress responses. Biochim Biophys Acta. 2012;1819(2): 86-96.

10. Puranik S, Sahu PP, Srivastava PS, Prasad M. NAC proteins: regulation and role in stress tolerance. Trends Plant Sci. 2012;17(6):369-81. 
11. Rushton DL, Tripathi P, Rabara RC, Lin J, Ringler P, Boken AK, Langum TJ, Smidt L, Boomsma DD, Emme NJ, et al. WRKY transcription factors: key components in abscisic acid signalling. Plant Biotechnol J. 2012;10(1):2-11.

12. Ariel FD, Manavella PA, Dezar CA, Chan RL. The true story of the HD-zip family. Trends Plant Sci. 2007;12(9):419-26.

13. Emanuelle $\mathrm{S}$, Hossain MI, Moller IE, Pedersen $\mathrm{HL}$, van de Meene AML, Doblin MS, Koay A, Oakhill JS, Scott JW, Willats WGT, et al. SnRK1 from Arabidopsis thaliana is an atypical AMPK. Plant J. 2015;82(2):183-92.

14. Crozet $P$, Jammes F, Valot B, Ambard-Bretteville F, Nessler S, Hodges M, Vidal J, Thomas M. Cross-phosphorylation between Arabidopsis thaliana sucrose nonfermenting 1-related protein kinase 1 (AtSnRK1) and its activating kinase (AtSnAK) determines their catalytic activities. J Biol Chem. 2010;285(16):12071-7.

15. McCartney RR, Schmidt MC. Regulation of Snf1 kinase: ACTIVATION REQUIRES PHOSPHORYLATION OF THREONINE 210 BY AN UPSTREAM KINASE AS WELL AS a DISTINCT STEP MEDIATED BY THE Snf4 SUBUNIT. J Biol Chem. 2001;276(39):36460-6.

16. Shen W, Reyes MI, Hanley-Bowdoin L. Arabidopsis protein kinases GRIK1 and GRIK2 specifically activate SnRK1 by phosphorylating its activation loop. Plant Physiol. 2009:150:996-1005.

17. Stein SC, Woods A, Jones NA, Davison MD, Carling D. The regulation of AMPactivated protein kinase by phosphorylation. Biochem J. 2000;345(3):437.

18. Baena-González E, Sheen J. Convergent energy and stress signaling. Trends Plant Sci. 2008;13(9):474-82.

19. Hong S-P, Carlson M. Regulation of Snf1 protein kinase in response to environmental stress. J Biol Chem. 2007;282(23):16838-45.

20. Cho Y-H, Hong J-W, Kim E-C, Yoo S-D. Regulatory functions of SnRK1 in stress-responsive gene expression and in plant growth and development. Plant Physiol. 2012;158(4):1955

21. Soto-Burgos J, Bassham DC. SnRK1 activates autophagy via the TOR signaling pathway in Arabidopsis thaliana. PLoS One. 2017;12(8):e0182591.

22. Coello P, Hey SJ, Halford NG. The sucrose non-fermenting-1-related (SnRK) family of protein kinases: potential for manipulation to improve stress tolerance and increase yield. J Exp Bot. 2010;62(3):883-93.

23. Liang S, Lu K, Wu Z, Jiang S-C, Yu Y-T, Bi C, Xin Q, Wang X-F, Zhang D-P. A link between magnesium-chelatase $\mathrm{H}$ subunit and sucrose nonfermenting 1 (SNF1)-related protein kinase SnRK2.6/OST1 in Arabidopsis guard cell signalling in response to abscisic acid. J Exp Bot. 2015;66(20):6355-69.

24. Chen L, Ren F, Zhou L, Wang Q-Q, Zhong H, Li X-B. The Brassica napus Calcineurin B-like 1/CBL-interacting protein kinase 6 (CBL1/CIPK6) component is involved in the plant response to abiotic stress and ABA signalling. J Exp Bot. 2012;63(17):6211-22.

25. Ohta M, Guo Y, Halfter U, Zhu J-K. A novel domain in the protein kinase SOS2 mediates interaction with the protein phosphatase 2C ABI2. Proc Natl Acad Sci. 2003;100(20):11771.

26. Rodrigues A, Adamo M, Crozet P, Margalha L, Confraria A, Martinho C, Elias A, Rabissi A, Lumbreras V, González-Guzmán M, et al. ABI1 and PP2CA phosphatases are negative regulators of Snf1-related protein Kinase1 signaling in Arabidopsis. Plant Cell. 2013;25(10):3871.

27. Yu Y, Fu J, Xu Y, Zhang J, Ren F, Zhao H, Tian S, Guo W, Tu X, Zhao J, et al. Genome re-sequencing reveals the evolutionary history of peach fruit edibility. Nat Commun. 2018:9(1):5404

28. Ashraf M, Foolad MR. Roles of glycine betaine and proline in improving plant abiotic stress resistance. Environ Exp Bot. 2007:59(2):206-16.

29. Yang Y, Tang N, Xian Z, Li Z. Two SnRK2 protein kinases genes play a negative regulatory role in the osmotic stress response in tomato. Plant Cell Tissue Organ Cult. 2015;122(2):421-34.

30. Love Ml, Huber W, Anders S. Moderated estimation of fold change and dispersion for RNA-seq data with DESeq2. Genome Biol. 2014;15(12):550.

31. Varet H, Brillet-Guéguen L, Coppée J-Y, Dillies M-A. SARTools: a DESeq2- and EdgeR-based R pipeline for comprehensive differential analysis of RNA-Seq data. PLoS One. 2016;11(6):e0157022.

32. Danquah A, de Zelicourt A, Colcombet J, Hirt H. The role of ABA and MAPK signaling pathways in plant abiotic stress responses. Biotechnol Adv. 2014; 32(1):40-52.

33. Wang P, Xue L, Batelli G, Lee S, Hou Y-J, Van Oosten MJ, Zhang H, Tao WA Zhu J-K. Quantitative phosphoproteomics identifies SnRK2 protein kinase substrates and reveals the effectors of abscisic acid action. Proc Natl Acad Sci. 2013;110(27):11205

34. Li R, Zhang J, Wei J, Wang H, Wang Y, Ma R. Functions and mechanisms of the CBL-CIPK signaling system in plant response to abiotic stress. Prog Nat Sci. 2009;19(6):667-76.
35. Im JH, Cho Y-H, Kim G-D, Kang G-H, Hong J-W, Yoo S-D. Inverse modulation of the energy sensor Snf1-related protein kinase 1 on hypoxia adaptation and salt stress tolerance in Arabidopsis thaliana. Plant Cell Environ. 2014; 37(10):2303-12.

36. Hwang H-H, Wang C-H, Huang H-W, Chiang C-P, Chi S-F, Huang F-C, Yen HE Functional analysis of MCSnRK1 (SNF1-related protein kinase 1) in regulating $\mathrm{Na} / \mathrm{K}$ homeostasis in transgenic cultured cells and roots of halophyte Mesembryanthemum crystallinum. Plant Cell Rep. 2019;38(8):915-26.

37. Polge C, Thomas M. SNF1/AMPK/SnRK1 kinases, global regulators at the heart of energy control? Trends Plant Sci. 2007;12(1):20-8.

38. You J, Chan Z. ROS Regulation During Abiotic Stress Responses in Crop Plants. Front Plant Sci. 2015;6:1092.

39. Miller GAD, Suzuki N, Ciftci-Yilmaz S, Mittler RON. Reactive oxygen species homeostasis and signalling during drought and salinity stresses. Plant Cell Environ. 2010:33(4):453-67.

40. Mittler R, Vanderauwera S, Gollery M, Van Breusegem F. Reactive oxygen gene network of plants. Trends Plant Sci. 2004:9(10):490-8.

41. Bedard K, Krause K-H. The NOX family of ROS-generating NADPH oxidases: physiology and pathophysiology. Physiol Rev. 2007;87(1):245-313.

42. Szymańska PK, Polkowska-Kowalczyk L, Lichocka M, Maszkowska J Dobrowolska G. SNF1-Related Protein Kinases SnRK2.4 and SnRK2.10 Modulate ROS Homeostasis in Plant Response to Salt Stress. Int J Mol Sci. 2019;20(1):143.

43. Zhao FG, Qin P. Protective effect of exogenous polyamines on root tonoplast function against salt stress in barley seedlings. Plant Growth Regul. 2004;42(2):97-103.

44. Liu J, Zhu JK. Proline accumulation and salt-stress-induced gene expression in a salt-hypersensitive mutant of Arabidopsis. Plant Physiol. 1997;114(2):591.

45. Yoshida T, Fujita Y, Maruyama K, Mogami J, Todaka D, Shinozaki K, Yamaguchi-Shinozaki K. Four Arabidopsis AREB/ABF transcription factors function predominantly in gene expression downstream of SnRK2 kinases in abscisic acid signalling in response to osmotic stress. Plant Cell Environ. 2015:38(1):35-49.

46. Wang P, Zhu J-K, Lang Z. Nitric oxide suppresses the inhibitory effect of abscisic acid on seed germination by S-nitrosylation of SnRK2 proteins. Plant Signal Behav. 2015;10(6):e1031939.

47. Yan S, Dong X. Perception of the plant immune signal salicylic acid. Curr Opin Plant Biol. 2014;20:64-8

48. Jossier M, Bouly J-P, Meimoun P, Arjmand A, Lessard P, Hawley S, Grahame Hardie D, Thomas M. SnRK1 (SNF1-related kinase 1) has a central role in sugar and ABA signalling in Arabidopsis thaliana. Plant J. 2009;59(2):316-28.

49. Dai D, Tong H, Cheng L, Peng F, Zhang T, Qi W, Song R. Maize Dek33 encodes a pyrimidine reductase in riboflavin biosynthesis that is essential for oil-body formation and ABA biosynthesis during seed development. J Exp Bot. 2019;70(19):5173-87.

50. Radchuk R, Radchuk V, Weschke W, Borisjuk L, Weber H. Repressing the expression of the SUCROSE NONFERMENTING-1-RELATED PROTEIN KINASE gene in pea embryo causes pleiotropic defects of maturation similar to an abscisic acid-insensitive phenotype. Plant Physiol. 2006;140(1):263-78.

51. Coello P, Hirano E, Hey SJ, Muttucumaru N, Martinez-Barajas E, Parry MA Halford NG. Evidence that abscisic acid promotes degradation of SNF1related protein kinase (SnRK) 1 in wheat and activation of a putative calcium-dependent SnRK2. J Exp Bot. 2012;63(2):913-24.

52. Olsen AN, Ernst HA, Leggio LL, Skriver K. NAC transcription factors: structurally distinct, functionally diverse. Trends Plant Sci. 2005;10(2):79-87.

53. Kato H, Motomura T, Komeda Y, Saito T, Kato A. Overexpression of the NAC transcription factor family gene ANAC036 results in a dwarf phenotype in Arabidopsis thaliana. J Plant Physiol. 2010;167(7):571-7.

54. Hong $Y$, Zhang H, Huang L, Li D, Song F. Overexpression of a stressresponsive NAC transcription factor gene ONAC022 improves drought and salt tolerance in Rice. Front Plant Sci. 2016;7:4.

55. Wu A, Allu AD, Garapati P, Siddiqui H, Dortay H, Zanor M-I, Asensi-Fabado MA, Munné-Bosch S, Antonio C, Tohge T, et al. JUNGBRUNNEN1, a reactive oxygen species-responsive NAC transcription factor, Regulates Longevity in Arabidopsis. Plant Cell. 2012;24(2):482.

56. Ebrahimian-Motlagh S, Ribone PA, Thirumalaikumar VP, Allu AD, Chan RL, Mueller-Roeber B, Balazadeh S. JUNGBRUNNEN1 Confers Drought Tolerance Downstream of the HD-Zip I Transcription Factor AtHB13. Front Plant Sci. 2017:8:2118.

57. Meng X, Wang J-R, Wang G-D, Liang X-Q, Li X-D, Meng Q-W. An R2R3-MYB gene, LeAN2, positively regulated the thermo-tolerance in transgenic tomato. J Plant Physiol. 2015;175:1-8. 
58. JEF RBH, Hoffmann NL, Wallroth M, Eichholtz D, Rogers SG, Fraley RT. A simple and general method for transferring genes into plants. Science. 1985;227(4691):1229.

59. Zhang Y, Primavesi LF, Jhurreea D, Andralojc PJ, Mitchell RA, Powers SJ, Schluepmann H, Delatte T, Wingler A, Paul MJ. Inhibition of SNF1-related protein kinase1 activity and regulation of metabolic pathways by trehalose6-phosphate. Plant Physiol. 2009;149(4):1860-71.

60. Bates LS, Waldren RP, Teare ID. Rapid determination of free proline for water-stress studies. Plant Soil. 1973;39(1):205-7.

61. Ma N-N, Zuo Y-Q, Liang X-Q, Yin B, Wang G-D, Meng Q-W. The multiple stress-responsive transcription factor SINAC1 improves the chilling tolerance of tomato. Physiol Plant. 2013;149(4):474-86

62. Hu D-G, Ma Q-J, Sun C-H, Sun M-H, You C-X, Hao Y-J. Overexpression of MdSOS2L1, a CIPK protein kinase, increases the antioxidant metabolites to enhance salt tolerance in apple and tomato. Physiol Plant. 2016;156(2):201-14.

63. Wang YC, Qu GZ, Li HY, Wu YJ, Wang C, Liu GF, Yang CP. Enhanced salt tolerance of transgenic poplar plants expressing a manganese superoxide dismutase from Tamarix androssowii. Mol Biol Rep. 2009;37(2):1119.

64. Aebi H. Catalase. In: Bergmeyer HU, editor. Methods of Enzymatic Analysis. 2nd ed. New York: Academic Press; 1974. p. 673-84.

65. Pütter J. Peroxidases. In: Bergmeyer HU, editor. Methods of Enzymatic Analysis. 2nd ed. New York: Academic Press; 1974. p. 685-90.

66. Wang $Y$, Jiang J, Zhao $X$, Liu G, Yang C, Zhan L. A novel LEA gene from Tamarix androssowii confers drought tolerance in transgenic tobacco. Plant Sci. 2006;171(6):655-62.

67. Jacyn Baker C, Mock NM. An improved method for monitoring cell death in cell suspension and leaf disc assays using Evans blue. Plant Cell Tissue Organ Cult. 1994;39(1):7-12.

\section{Publisher's Note}

Springer Nature remains neutral with regard to jurisdictional claims in published maps and institutional affiliations.

Ready to submit your research? Choose BMC and benefit from:

- fast, convenient online submission

- thorough peer review by experienced researchers in your field

- rapid publication on acceptance

- support for research data, including large and complex data types

- gold Open Access which fosters wider collaboration and increased citations

- maximum visibility for your research: over $100 \mathrm{M}$ website views per year

At $\mathrm{BMC}$, research is always in progress.

Learn more biomedcentral.com/submissions 